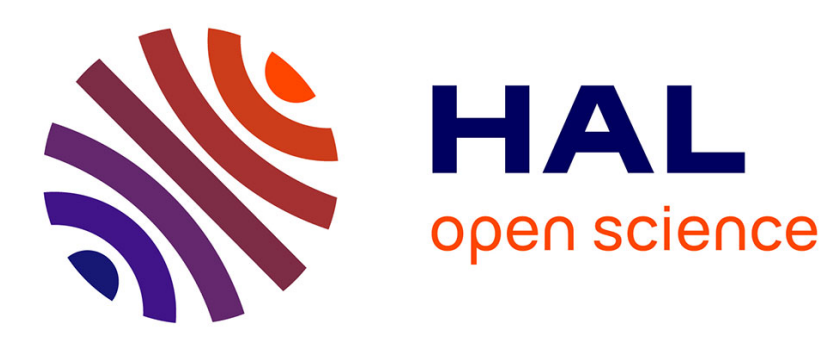

\title{
Topologic and electronic density driven generation of alkali cation complexes
}

Hamza Boufroura, Salomé Poyer, Anne Gaucher, Cécile Huin, Jean-Yves

Salpin, Gilles Clavier, Damien Prim

\section{- To cite this version:}

Hamza Boufroura, Salomé Poyer, Anne Gaucher, Cécile Huin, Jean-Yves Salpin, et al.. Topologic and electronic density driven generation of alkali cation complexes. Chemistry - A European Journal, 2018, 24 (34), pp.8656-8663. 10.1002/chem.201800707 . hal-01890491

\section{HAL Id: hal-01890491 \\ https://hal.science/hal-01890491}

Submitted on 8 Oct 2018

HAL is a multi-disciplinary open access archive for the deposit and dissemination of scientific research documents, whether they are published or not. The documents may come from teaching and research institutions in France or abroad, or from public or private research centers.
L'archive ouverte pluridisciplinaire $\mathbf{H A L}$, est destinée au dépôt et à la diffusion de documents scientifiques de niveau recherche, publiés ou non, émanant des établissements d'enseignement et de recherche français ou étrangers, des laboratoires publics ou privés. 


\title{
Topologic and electronic density driven generation of alkali cation complexes.
}

\author{
Hamza Boufroura, ${ }^{[a]}$ Salomé Poyer, ${ }^{[b, c]}$ Anne Gaucher, ${ }^{[a]}$ Cécile Huin, ${ }^{[b, c]}$ Jean-Yves Salpin,,${ }^{[b, c]}$ Gilles \\ Clavier, ${ }^{[\mathrm{d}]}$ and Damien Prim ${ }^{\star[\mathrm{a}]}$
}

\begin{abstract}
The formation and characterization of $\mathrm{K}^{+}-$and $\mathrm{Cs}^{+}-$ complexes originating from the cooperativity of three non-covalent interactions is explored. The tridimensional preorganization of the naphthothiophene platform displays a favourable well-defined bay region combining a $\pi$ fragment and a carbonyl moiety flanking a central sulfur atom. A joint theoretical and experimental IRMPD study allowed deciphering the key contribution of the orthogonal phenyl fragment to the elaboration of alkali metal complexes. In combination with $\mathrm{S}$ - and CO-interactions, the $\pi$-cation interaction significantly enhances the binding energies of naphthothiophene derivatives.
\end{abstract}

\section{Introduction}

The topology of a molecular architecture is set by both covalent bonding network and noncovalent interactions. Devising strategies allowing to shape, modify or restore privileged conformations and thus modulate innate properties of a chemical structure is of continuing interest for a broad scientific community. In the aromatic series, the way in which aromatic interactions (e.g. $\pi-\pi$ or ion- $\pi$ ) in combination with repulsive steric clashes forge the three-dimensional molecular geometries is fascinating and conditions their applications in nanotechnology, material chemistry and life sciences. ${ }^{[1]}$ Thanks to steric and/or electronic contributions, planar aromatics can escape the flat world and serve as pivotal molecular platforms for the construction of 3-D complex single molecules as well as self-assembled architectures. ${ }^{[2]}$ Among them, the smallest elemental polycyclic aromatic building block, naphthalene, represents a useful model platform. Naphtalene is able to adapt its topology depending on its substitution pattern (figure 1). This unique spatial arrangement is at the origin of the binaphthyl chiral inducing and templating properties which have been widely used in various domains. ${ }^{[3]}$ The presence of two aromatic rings installed at peri positions confers on 1,8diarylnaphthalenes a peculiar twisted topology, ${ }^{[4]}$ which is based on two main features that are a cofacial orientation of these two

[a] Dr. H. Boufroura, Dr. A. Gaucher, Pr. Dr. D. Prim ILV, UVSQ, CNRS, Université Paris-Saclay, 78035 Versailles, France

E-mail: damien.prim@uvsq.fr

[b] Dr. S. Poyer, Dr. C. Huin, Dr. J.-Y. Salpin

LAMBE, Univ Evry, CNRS, CEA, Université Paris-Saclay, 91025 Evry, France.

[c] Dr. S. Poyer, Dr. C. Huin, Dr. J.-Y. Salpin

LAMBE, U-Cergy, Université Paris-Seine, 91025 Evry, France

[d] Dr. G. Clavier

PPSM, ENS Cachan, CNRS, Université Paris-Saclay, 94235

Cachan, France

Supporting information for this article is given via a link at the end of the document.((Please delete this text if not appropriate)) aromatic groups, and their layout almost perpendicular to the naphthalene platform. The significant increase in steric congestion results in the deformation of the naphthalene backbone (figure 1).

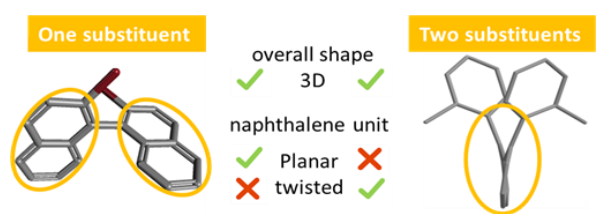

Figure 1. Impact of the substitution pattern on the 3D shape of naphthalene platforms.

The 1,8-diarylnaphthalenes have found applications as potential sensors and switches and been explored in terms of model systems to get insights into $\pi-\pi$ interactions and precursors for the synthesis of helical architectures. ${ }^{[5]}$

The replacement of a carbon atom by a heteroatom in these polycyclic aromatic architectures is likely to (i) generate the increase or the emergence of properties when considered as dopant in graphene derivatives, ${ }^{[6]}$ (ii) impact their spatial shape and structural parameters through steric effects. ${ }^{[7]}$ In this context, 9-arylnaphthothiophene represents a target which displays one phenyl ring and a condensed thiophene ring. As shown in Figure 2, this new platform differs from the parent 1,8 diarylnaphtalenes, binaphthyls and naphthothiophenes by the substitution pattern of the common naphthalene skeleton.

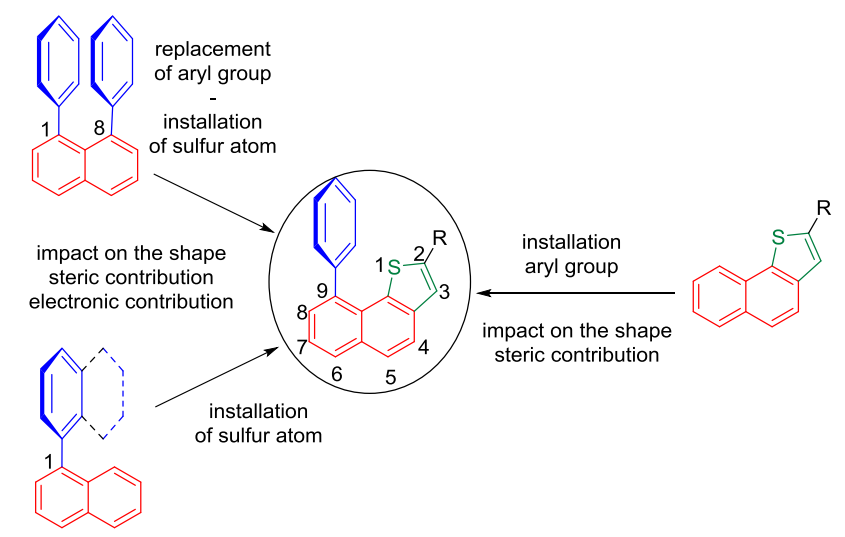

Figure 2. A new molecular platform at the crossroads of three different naphthalene-based motifs. 
In the 1,8-diarylnaphtalene, the replacement of one aryl group by the thiophene and thus the respective spatial arrangement between the phenyl ring and the sulfur atom ring are likely to impact the molecular shape of the architecture. A similar impact is foreseen when an additional heterocycle is installed in monosubstituted naphthalenes analogues of binaphthyls. ${ }^{[8]}$ In these series, the presence of the heteroatom within the molecule might also impact the electronic distribution on the naphthalene platform. Further, the selective installation of an aryl group in position 9 of the rigid and planar naphthothiophene unit will plausibly also induce modifications of its shape. The additional steric contribution might as well impact the properties of such molecules. Indeed, naphthothiophenes in $\pi$-conjugated molecular or polymeric systems display potential photoinduced electron transfer applications. ${ }^{[9]}$

9-Arylnaphthothiophene represents a new molecular platform at the crossroads of three different naphthalene-based motifs in terms of steric and electronic contributions. In this paper we describe first, the synthesis of a new molecular scaffold based on a 9-arylnaphthothiophene core and an additional $\pi$ fragment (Figure 3). The impact of the joint presence of the phenyl group located in position 9 of the molecule and the central sulfur atom on the shape of the architecture, is examined by means of theoretical calculations. Further, the presence of the sulfur atom at the heart of the molecule raises the question of its electronic contribution to the newly-formed bay region. Second, the comparison of fully aromatic and partially hydrogenated platforms (based on a dihydronaphthalene fragment) on the deformation or restoration of the naphthalene shape, and on the electronic contribution to the bay region leaned at a naphthalene platform, is given. Third, the ability of the favorable spatial arrangement and electronic environment of the fully aromatic and partially hydrogenated backbones to generate $\mathrm{K}^{+}$- and $\mathrm{Cs}^{+}-$ complexes that originate from a combination of three non covalent weak interactions including S-, $\mathrm{CO}$ - and $\pi$-interactions is explored (Figure 3). Indeed, the $\pi$-donor ability of sulfur toward alkali metals has already been proven and the study of alkali metal cation- $\pi$ interactions on new model ligands is a crucial area in biological systems as nearly $10 \%$ of amino acid side chains contain an aromatic ring. ${ }^{[10]}$ Moreover, potassiumselective channel represents the largest and the most diverse group of ion channels. As recently discovered, channel mutations, responsible of diseases called channelopathies, [11] lead to neuromuscular disorders and heart diseases. Designing single molecule models ${ }^{[12]}$ combined with computational and analytical studies could be insightful for a better understanding on how cation-non covalent weak interactions are involved in structural and functional roles, such as controlling protein conformation and molecular recognition. ${ }^{[13]}$ Impact of the presence of the phenyl and the ester group on binding energies is finally discussed.

Synthesis Steric and electronic contribution Complexation

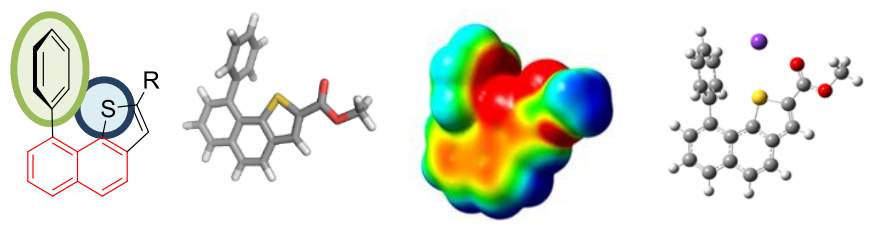

Figure 3. Synthesis, steric and electronic contribution and complexation of naphthothiophene platforms

\section{Results and Discussion}

Synthesis of 9-phenylnaphthothiophene 1 was achieved using a short sequence involving the construction of (dihydro)naphthothiophene derivative 2 from commercially available bromotetralone in three steps and $98 \%$ yield (scheme 1). ${ }^{[14]}$ Aromatisation to obtain the naphthalene platform 3 in $76 \%$ yield was achieved using DDQ in refluxing toluene. The last step was dedicated to the installation of the phenyl group. To this end, phenylboronic acid was reacted with bromoderivative $\mathbf{3}$ under Suzuki cross-coupling conditions. The use of $\mathrm{Pd}(\mathrm{OAc})_{2}(10 \%)$, SPhos (20\%) and CsF (4eq.) allowed the preparation of target 1 in an almost quantitative yield.

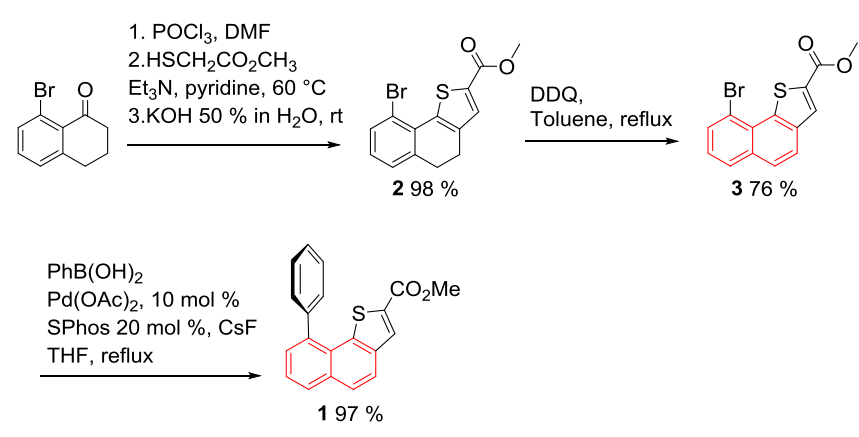

Scheme 1. Synthesis of naphthothiophene 1.

The geometry of compound 1 was studied at the B3LYP/6$31+G(d, p)$ level of theory without any solvent correction (Figure 4). Harmonic vibrational frequencies were calculated to characterize the stationary points as a local minima. 1 overall shape is organized around two almost perpendicular planes described first by the naphthalene platform and second by the phenyl ring (blue) as confirmed by the $88^{\circ} \mathrm{Ph}_{1}-\mathrm{Ph}_{2}$ dihedral angles. Two additional features are indicative of the spatial arrangement within the architecture of compound 1. Interestingly, $\mathrm{Ph}_{1}$ (blue) faces the thiophene sulfur atom and displays a $\mathrm{S}-\mathrm{Ph}_{1}$ centroid distance of $3.44 \AA$. The presence of the almost perpendicular phenyl ring has only a minor impact on the $\mathrm{Ph}_{2}-\mathrm{Th}$ dihedral angle which represents the deformation of the overall platform.
a)
b)
c)
d) 
(1)
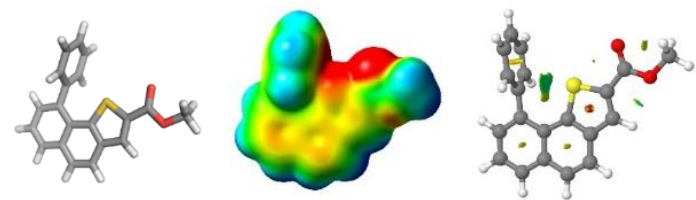

Figure 4. Spatial arrangement of compound 1 (a, b), ESP map (c, red = negative and blue $=$ positive) and NCIplot (e).

As shown by ESP (Electrostatic potential surfaces) map (Figure 4c), the three-dimensional spatial arrangement of the sulfur central atom surrounded by both the aromatic $\mathrm{Ph}_{1}$ and the carbonyl fragment contributes to a well-defined electronically rich bay-region. The syn conformation between the thiophene and the carbonyl moiety observed is in complete agreement with recent reports, ${ }^{[15]}$ highlighting the key role played by the sulfur atom in both the stabilization of the 3D-shape and the enhancement of the electronic density within the bay-region. Further Natural Bond Orbital (NBO) calculations confirmed the intervention of the sulfur atom in the stabilization of the overall architecture, evidenced by delocalization between (i) the oxygen sp lone pair into the sulfur atom $\mathrm{d}$ orbitals and (ii) of $\pi \mathrm{Ph}_{1}$ orbitals into the sulfur $d$ orbitals. NClplot (Non Covalent Interaction plotting) also shows a favorable non-covalent interaction between $\mathrm{Ph}_{1}$ and $\mathrm{S}$ (Figure 4d). The latter stabilizing interaction consequently shortens the distance between the sulfur atom and the phenyl ring centroid and contributes to the apparent lack of electronic repulsion by comparison with diarylnaphthalene. Both these points explain the small deformation of the naphthalene backbone.

In order to see if the $\mathrm{Ph}_{1}-\mathrm{Ph}_{2}, \mathrm{Ph}_{2}$-Th dihedral angles and the S$\mathrm{Ph}_{1}$ centroid distance are strongly depending on the deformation or rigidity of the naphthalene platform, we decided to synthesize the and compare the 3D-shape of the partially hydrogenated analogue 4. The latter was obtained using the same methodology starting from (dihydro)-naphthothiophene 2 and cross coupling reaction (ESI). Similarly to compound $\mathbf{1 , 4}$ is organized around the same two planes between the blue and red phenyl rings. $\mathrm{Ph}_{1}-\mathrm{Ph}_{2}$ dihedral angle decreases from 88 to $69^{\circ}$ which can be attributed to a release of steric strain that accompanies the increased flexibility of the (dihydro)naphthalene backbone (figure 5). As expected moving from the more constrained and rigid aromatic platform 1 to the distorted partially hydrogenated 4 , impacts the $\mathrm{Ph}_{2}$-Th dihedral angle which raises to $20^{\circ}$. In contrast the twisted shape of the naphthalene platform in 4, has only a minor effect on the $\mathrm{S}-\mathrm{Ph}_{1}$ centroid distances $\left(3.44 \AA\right.$ vs $3.36 \AA$ ). Further, $\mathrm{Ph}_{1}$ (blue) still faces the thiophene sulfur atom. As shown by ESP map (Figure $5 c$ ), the three-dimensional spatial arrangement of the overall architecture remains almost similar and contributes again to a well-defined electronically rich bay-region. Natural Bond Orbital (NBO) calculations evidenced a similar stabilizing effect of the overall architecture through the delocalization between (i) the oxygen sp lone pair into the sulfur atom $d$ orbitals and (ii) of $\pi \mathrm{Ph}_{1}$ orbitals into the sulfur d orbitals. NClplot (Figure $5 \mathrm{~d}$ ) also shows a favorable non-covalent interaction between $\mathrm{Ph}_{1}$ and $\mathrm{S}$ in 4 . In both the fully aromatic and partially hydrogenated series, the sulfur atom undoubtedly plays a major role in both the stabilization of the 3D-shape and the enhancement of the electronic density within the bay-region. a)

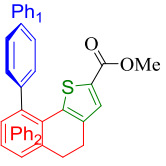

b)

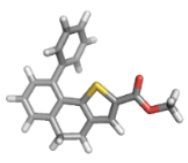

c)

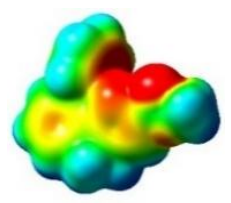

d)

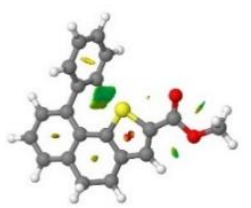

Figure 5. Spatial arrangement of compound 4 (a, b), ESP map (c, red = negative and blue $=$ positive) and NCIplot (e).

We next examined and compared the ability of ligands $\mathbf{1}$ and $\mathbf{4}$ to host alkali cations. To perform this study, we combined theoretical DFT calculations and IRMPD spectra of the electrospray-generated complexes of neutral molecule-cation. IRMPD spectroscopy of mass-selected ions is established as a powerful approach for the structural characterization of gaseous ions and has been successfully applied to the characterization of neutral molecule-ion complexes. ${ }^{[16]}$ The complexes with $\mathrm{K}^{+}$were optimized using B3LYP ${ }^{[17]}$ or M06-2X ${ }^{[18]}$ density functional and $6-31+G(d, p)$ basis set implemented in the Gaussian 09 software and further analyzed with an energy calculation with the $311++G(d, p)$ basis set. The basis set superposition error (BSSE) was corrected using the counterpoise method. As shown in Figure 6, well defined multiple interactions account for the formation of complexes $\mathbf{1}-\mathbf{K}^{+}$and $\mathbf{4}-\mathbf{K}^{+}$.
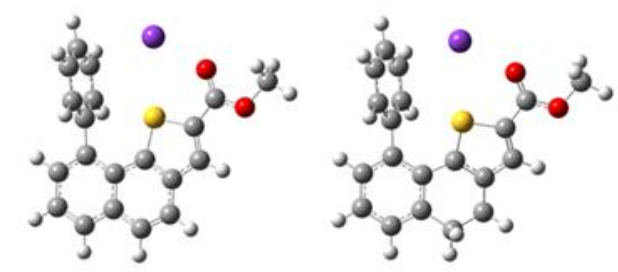

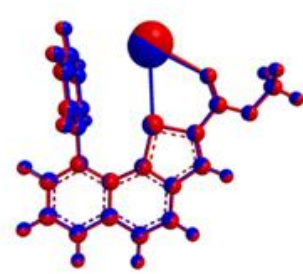

$1-\mathrm{K}^{+}$

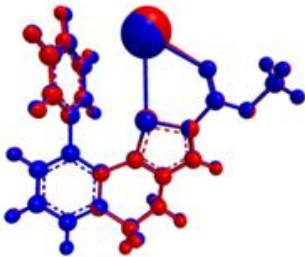

4-K $\mathrm{K}^{+}$
Figure 6. Computed structures of $\mathbf{1}-\mathrm{K}^{+}$and $\mathbf{4 - \mathbf { K } ^ { + }}$ (top) and overlay of structures obtained by B3LYP (red) and M06-2X (blue) methods (bottom).

Indeed, the structure of $\mathbf{1 - K ^ { + }}$ and $4-\mathbf{K}^{+}$complexes are set by three contributive $\mathrm{S}-\mathrm{K}^{+}, \mathrm{O}-\mathrm{K}^{+}$and $\pi-\mathrm{K}^{+}$interactions (structures 1$\mathbf{K}^{+}$and $\mathbf{4}-\mathbf{K}^{+}$, respectively). In both complexes, the alkali cation is located within the electronically rich bay-region (Figure 6, top). 
The 3D shapes of both architectures are very close regardless of the rigidity of the ligand and the method used (B3LYP or M06$2 \mathrm{X}$ ) as evidenced by overlays in Figure 6 (bottom). Therefore, only the B3LYP method was used subsequently. Other coordination schemes have been considered during our DFT study (See Figure $\mathrm{S} 11$ of the $\mathrm{SI}$ ), all the resulting structures being sensibly higher in energy.

Distances between contributive actors of the formation of complexes $\mathbf{1}-\mathbf{K}^{+}$and $\mathbf{4}-\mathbf{K}^{+}$are compiled in Table 1 for the global minimum, namely $\mathbf{1}-\mathbf{K}^{+}-\mathbf{a}$ and $\mathbf{4}-\mathbf{K}^{+}-\mathbf{a}$, respectively. For each complex, distances decreased significantly from the largest $\pi-\mathrm{K}^{+}$ to the shortest $\mathrm{O}-\mathrm{K}^{+}$, suggesting a pivotal role of the carbonyl- $\mathrm{K}^{+}$ interaction. The formation of $\mathrm{K}^{+}$complexes within the bay-region arising from a triple interaction appears the most stable by comparison with other hypothetical complexes (Figure S11).

Table 1. B3LYP computed $S-M^{+}, O-M^{+}$and $\pi-M^{+}$distances (Å) for the complexes

\begin{tabular}{|c|c|c|c|c|}
\hline Entry & Complexes & $\boldsymbol{\Pi}-\mathbf{M}^{+[a]}$ & $S-M^{+}$ & $O-M^{+}$ \\
\hline 1 & $1-\mathrm{K}^{+}$ & 3.684 & 3.273 & 2.635 \\
\hline 2 & $5-K^{+}\left(\Delta 1-K^{+} / 5-K^{+}\right)$ & - & $3.879(-0.606)$ & 2.525 (0.110) \\
\hline 3 & $7-K^{+}\left(\Delta 1-K^{+} / 7-K^{+}\right)$ & $3.115(0.569)$ & $3.115(0.569)$ & - \\
\hline 4 & $4-\mathrm{K}^{+}$ & 3.663 & 3.296 & 2.629 \\
\hline 5 & $6-K^{+}\left(\Delta 2-K^{+} / 6-K^{+}\right)$ & - & $4.091(-0.795)$ & $2.509(0.120)$ \\
\hline 6 & $8-K^{+}\left(\Delta 2-K^{+} / 8-K^{+}\right)$ & $2.989(0.674)$ & $3.189(0.107)$ & - \\
\hline 7 & $1-\mathrm{Cs}^{+}$ & 4.234 & 3.902 & 3.038 \\
\hline 8 & 4-Cs ${ }^{+}$ & 4.243 & 3.864 & 3.050 \\
\hline
\end{tabular}

[a] the distance was measured between the cation and the phenyl (Ph1) centroid

Analysis of the results strongly supports the important contributions from the $\pi$-cloud of $\mathrm{Ph}_{1}$, the $\mathrm{sp}^{2}$ lone pair of $\mathrm{S}$ atom and $\mathrm{sp}$ lone pair of $\mathrm{O}$ atom from the carbonyl fragment (in increasing order of importance from NBO analysis) in the formation of the complexes. In other words, the main contributions to the formation of the complex come from the interactions of the potassium with the $\mathrm{O}$ and $\mathrm{S}$ atoms, the $\mathrm{Ph}_{1}$ acting as an additional stabilizing force. NClplot allows to visualize all three contributions (Table 2 entry 1 ).

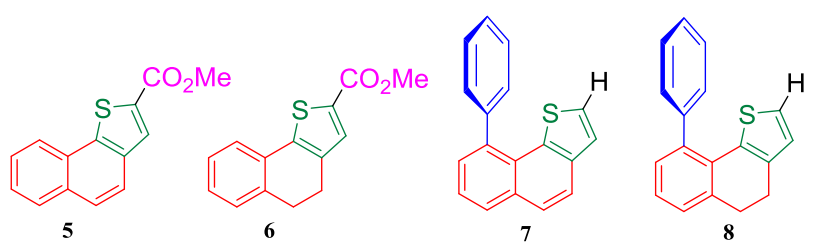

Figure 7. Model ligands 5 - 8.

Table 2. $\mathrm{NCl}$ plot and calculated binding energies (BE in $\mathrm{kJ} / \mathrm{mol}$ )

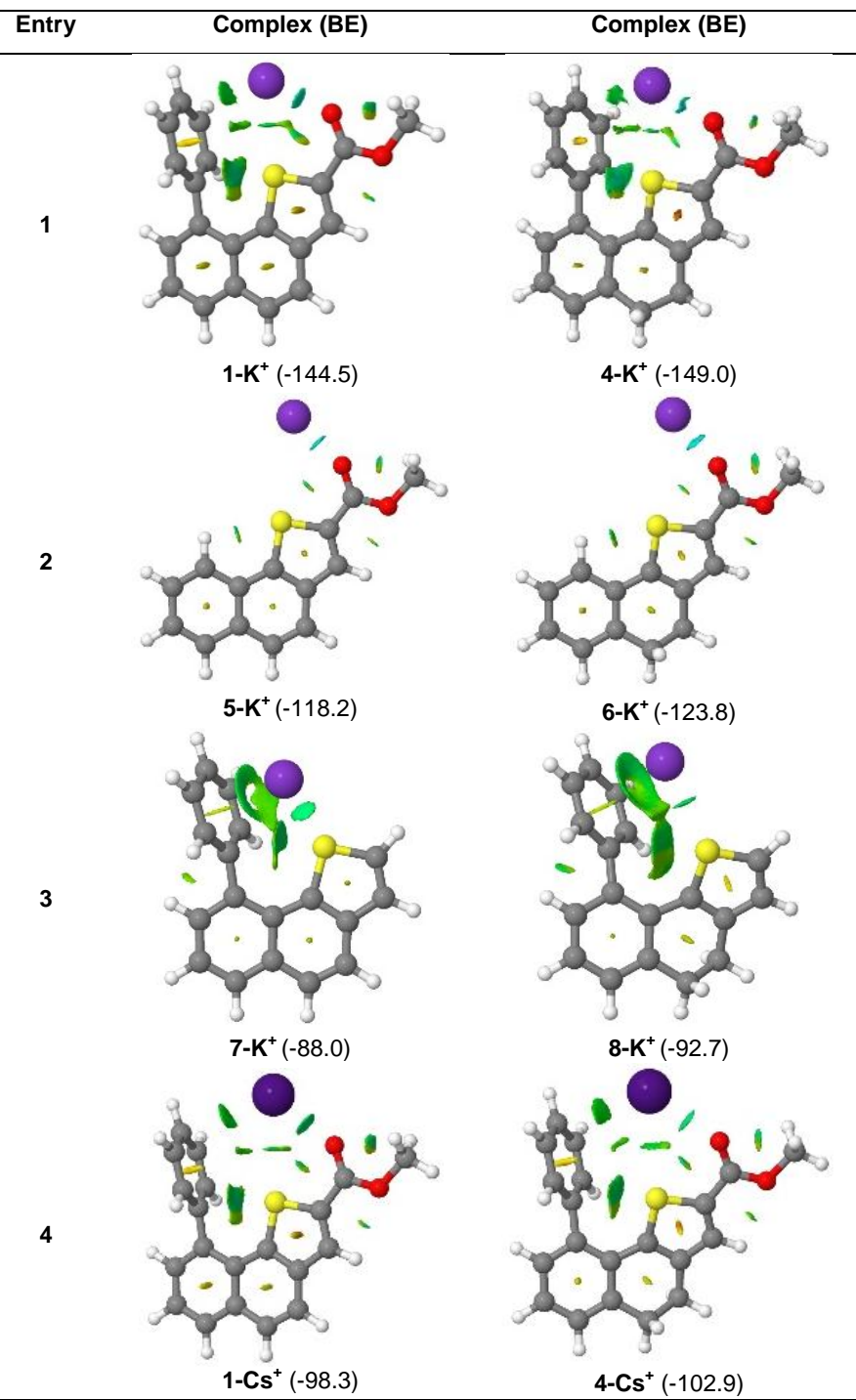

Model ligands 5 and 6 where the $\mathrm{Ph}_{1}$ ring was omitted and 7 and 8 where the ester group was removed (Figure 7), have been calculated as well as their $\mathrm{K}^{+}$complexes in order to prove the key role of those fragments. In the absence of the side phenyl ring, the $\mathrm{O}-\mathrm{K}^{+}$distances are shortened by $0.11-0.12 \AA$ while the $\mathrm{S}-\mathrm{K}^{+}$distances are lengthened by $0.6-0.8 \AA$ (Table 1 ) compared to 1 and 4. Thus, when removing $\mathrm{Ph}_{1}$ ring, the 
potassium cation interacts essentially with the sp lone pair of the oxygen atom of the carbonyl. The NClplots (Table 2, entry 2) display an attractive interaction between the potassium and the oxygen of the carbonyl but none with the sulfur. In the absence of the ester moiety, the $\pi-\mathrm{M}^{+}$distances are much shorter than in the parent complexes $1-\mathbf{K}^{+}$and $\mathbf{4}-\mathbf{K}^{+}$, by 0.57 and $0.67 \AA$ in 7 $\mathbf{K}^{+}$and $\mathbf{8}-\mathbf{K}^{+}$, respectively. The $\mathbf{S}-\mathrm{K}^{+}$distances are also shortened but to a lesser extent $(0.07$ and $0.1 \AA)$. The $\mathrm{NCl}$ plots of those complexes (Table 2, entry 3 ) highlight an extended zone of interaction between the phenyl ring and the potassium. It can thus be concluded that the side phenyl ring plays an important role in the complex formation since in its absence, the ester is the only interacting element. Conversely when the ester is removed, the potassium cation moves towards the phenyl ring to establish a stronger interaction.

The primary role of the ester in the formation of the complex is also apparent from the decreased binding energy for complexes 7- $\mathrm{K}^{+}$and $\mathbf{8}-\mathrm{K}^{+}$compared to $\mathbf{1 - \mathrm { K } ^ { + }}$ and $\mathbf{4}-\mathrm{K}^{+}$by nearly $56 \mathrm{~kJ} / \mathrm{mol}$ (Table 2). But the important role of the $\mathrm{Ph}_{1}$ ring as additional binding site in $\mathbf{1}$ and $\mathbf{4}$ for the formation of stable complexes with $\mathrm{K}^{+}$is seen from the increased interaction energy for those ligands compared to 5 and 6 by approximately $26 \mathrm{~kJ} / \mathrm{mol}$.

It is also noteworthy that the structural modification from $\mathbf{1}$ to $\mathbf{4}$ (naphthothiophene vs (dihydro)-naphthothiophene) has a small influence on the calculated binding energies (Table 2, entry 1) since it is $4.5 \mathrm{~kJ} / \mathrm{mol}$ smaller for $\mathbf{1}-\mathbf{K}^{+}$than for $\mathbf{4}-\mathbf{K}^{+}$. Another factor influencing the formation of stable complexes is reorganization of the ligand. In the case of $\mathbf{1}$ and $\mathbf{4}$, calculations have shown that there is a well-defined electronically rich bayregion that can accommodate a $\mathrm{K}^{+}$(Figures 4 and 5 , respectively). The good compatibility between the two partners is also evidenced by the small deformation energies ${ }^{[19]}$ calculated for the ligands upon going from their free to complex geometries (Table 3). The minimal geometrical reorganization of the ligands can be clearly seen from the overlap of the structures (Figure 8).

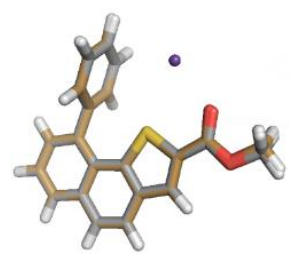

$\mathbf{1} / / \mathbf{1}-\mathrm{K}^{+} \mathrm{RMS}$ over 20 heavy atoms $=0.0021 \AA$

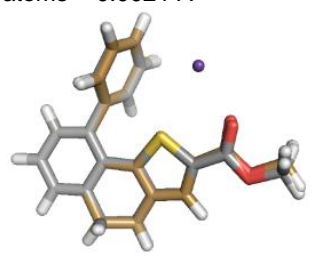

4//4-K $\mathrm{K}^{+} \mathrm{RMS}$ over 20 heavy atoms $=0.0066 \AA$

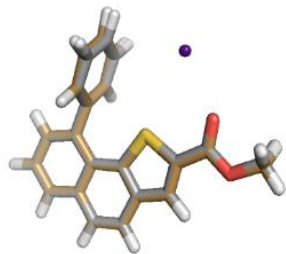

1//1-Cs ${ }^{+}$RMS over 20 heavy atoms $=0.073 \AA$

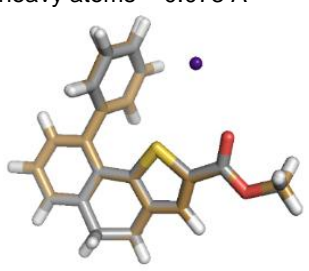

4//4-Cs ${ }^{+}$RMS over 20 heavy atoms $=0.050 \AA$
Figure 8. Overlay of optimized ligand and complexes (B3LYP/6-31+G(d,p)) Full details in figure S16.

Table 3. Deformation energies (in $\mathrm{kJ} / \mathrm{mol}$ ) for $\mathrm{K}^{+}$complexes of compounds 1, 4, 5 and $\mathbf{6}$ and $\mathrm{Cs}^{+}$complexes of compounds $\mathbf{1}$ and $\mathbf{4}$.

\begin{tabular}{cc}
\hline Complexe & Deformation energy \\
\hline $\mathbf{1}-\mathbf{K}^{\mathbf{2}}$ & 6.9 \\
$\mathbf{1 - C \mathbf { S } ^ { + }}$ & 9.7 \\
$\mathbf{5}-\mathbf{K}^{+}$ & 4.5 \\
$\mathbf{7}-\mathbf{K}^{+}$ & 5.5 \\
$\mathbf{4}-\mathbf{K}^{+}$ & 8.1 \\
$\mathbf{4}-\mathbf{C s}^{+}$ & 8.9 \\
$\mathbf{6}-\mathbf{K}^{+}$ & 5.0 \\
$\mathbf{8}-\mathbf{K}^{+}$ & 5.3
\end{tabular}

The infrared absorption spectra of the most stable structure 1 and $4-\mathrm{K}^{+}$were calculated within the harmonic approximation. A scaling factor value of 0.98 has been applied in the 900-1800 $\mathrm{cm}^{-1}$ frequency range. IRMPD experiments on both $\mathbf{1 - \mathrm { K } ^ { + }}$ and $4-$ $\mathrm{K}^{+}$ions have been performed in the same spectral region, using a free electron laser (FEL) at the CLIO (Center Laser Infrarouge d'Orsay) facility. The IRMPD spectra are obtained by plotting the photofragmentation yield $\mathrm{R}\left(\mathrm{R}=-\ln \left[I_{\text {precursor }} /\left(I_{\text {precursor }}+\sum l_{\text {fragment }}\right)\right]\right.$ where $I_{\text {precursor }}$ and $I_{\text {fragment }}$ are the integrated intensities of the mass peaks of the precursor and the fragment ions, respectively) as a function of the frequency of the IR radiation. ${ }^{14}$ Finally, to be consistent with the experimental spectral resolution, the calculated spectra have been convoluted by a Lorentzian function with a FWHM of $15 \mathrm{~cm}^{-1}$ (experimental details are given in the SI). The photo-fragment observed by IRMPD is similar for both complex and corresponds to the formation of $\mathrm{K}^{+}$(loss of the intact ligand).

As shown in Figure 9, the experimental IRMPD spectra of the two complexes are very similar, suggesting the same binding scheme. The spectra indeed share an overwhelming feature around $1330 \mathrm{~cm}^{-1}$, an intense band around $1645 \mathrm{~cm}^{-1}$, and bands detected slightly below 1100 and $1200 \mathrm{~cm}^{-1}$. The region between $1400 \mathrm{~cm}^{-1}$ and $1450 \mathrm{~cm}^{-1}$ differs slightly in terms of band intensity and a small signal at about $1490 \mathrm{~cm}^{-1}$ is only observed for the $\mathbf{4}-\mathrm{K}^{+}$complex. The assignment of the IRMPD spectrum of the various complexes is based on the comparison with the spectra computed for the low energy-lying structures, and as illustrated by Figure 3, the harmonic computed spectra (gray) computed for the global minima, are globally in very good agreement with the experimental data (red). Almost all the IRMPD bands can be assigned by considering the IR active 
modes of the $\mathbf{1}-\mathbf{K}^{+}$and $\mathbf{4}-\mathbf{K}^{+}$structures, which are summarized in Table $\mathrm{S} 1$ and $\mathrm{S} 2$ of the Supporting Information, respectively. The other structures considered do not give a good agreement with experiments (see $\mathrm{SI}$ ). The intense band detected around $1645 \mathrm{~cm}^{-1}$ can be attributed to the $\mathrm{C}=\mathrm{O}$ stretch of the ester function. This $\mathrm{C}=\mathrm{O}$ stretch is significantly red-shifted with respect to the values typically encountered for unpertubated carbonyl groups $\left(\sim 1750-1800 \mathrm{~cm}^{-1}\right)$, and therefore indicates that this group presently interacts with the metallic centre. For each complex, several vibrational modes can account for the dominating experimental signal centered around $1330 \mathrm{~cm}^{-1}$, and notably the very intense $\mathrm{C} 2-\mathrm{O} 1$ stretching mode (see $\mathrm{SI}$ for atom numbering), computed at 1324 and $1328 \mathrm{~cm}^{-1}$ for $\mathbf{1}-\mathbf{K}^{+}$and $\mathbf{4}-\mathbf{K}^{+}$, respectively.

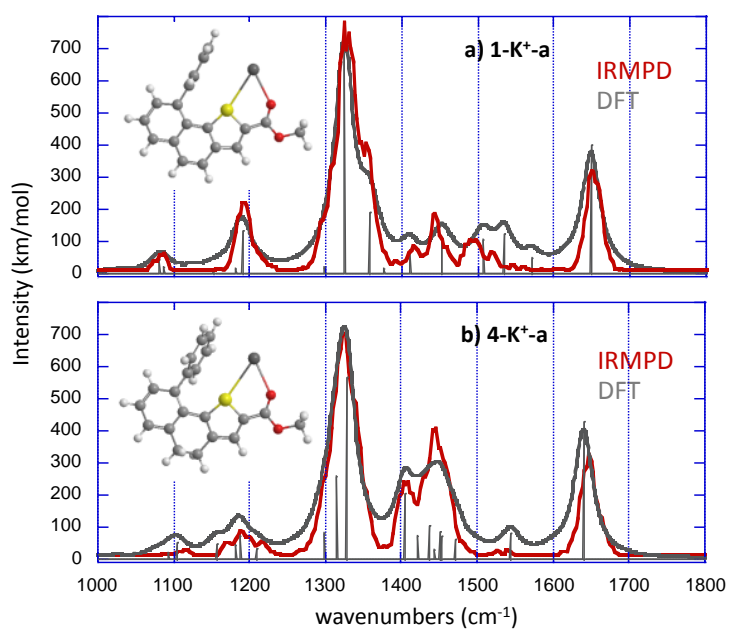

Figure 9. Overlay of experimental and computed infrared absorption spectra for a) $\mathbf{1 - K ^ { + }}$ and b) $\mathbf{4 - K ^ { + }}$. IRMPD irradiation time was set to $250-350 \mathrm{~ms}$.

Other bands are also computed in this region, such as twisting modes of the $\mathrm{CH}_{2}$ groups for $\mathbf{4}-\mathbf{K}^{+}$, or the naphthothiophene moiety breathing for both systems. Note also that the shoulder experimentally observed on the blue side of the band for the 1$\mathrm{K}^{+}$complex is also reproduced and can be ascribed to the stretching of aromatics $\mathrm{C}-\mathrm{C}$ bonds and notably $\mathrm{C}_{5}-\mathrm{C}_{7}$ and $\mathrm{C}_{8}-\mathrm{C}_{9}$. The experimental signal detected around $1190 \mathrm{~cm}^{-1}$ is mostly due to the $\mathrm{CH}_{3}$ wagging mode and out of plane deformations of $\mathrm{C}-\mathrm{H}$ and/or $\mathrm{CH}_{2}$ groups, whereas the band observed around $1100 \mathrm{~cm}^{-1}$ is associated with bending modes of aromatic $\mathrm{CH}$ groups. Note also that more bands are computed between 1400 and $1500 \mathrm{~cm}^{-1}$ for the $4-\mathrm{K}^{+}$structure, which may explain the more intense signals observed experimentally for this complex. These additional bands are associated with $\mathrm{CH}_{2}$ scissoring deformations of the saturated $\mathrm{CH}_{2}$ groups of the dihydrogenated ring.

To summarize, the full correlation between experimental and computed data confirmed the intervention and cooperatively of S-, O- and $\pi-\mathrm{K}+$ interactions to set the formation of $\mathbf{4 -}$ and $\mathbf{1 - \mathrm { K } ^ { + }}$ complexes. The ability of ligands $\mathbf{1}$ and $\mathbf{4}$ to host $\mathrm{Cs}^{+}$was next examined using a similar combined experimental and computed approach. In order to describe the alkali metal cation $\mathrm{Cs}^{+}$, we used the Los Alamos effective core potential (ECP) in combination with LAND2LZ basis set. ${ }^{[20]}$ IRMPD spectra obtained for both $\mathbf{4}-\mathbf{C s}^{+}$and $\mathbf{1 - C \mathbf { s } ^ { + }}$ are given in the Supporting Information (Figure S14) and turned out to be remarkably similar to those obtained with $\mathrm{K}^{+}$, again suggesting the combined interaction with the carbonyl group, the sulfur atom and the phenyl ring. Given this similarity, we restricted our calculations to the structure corresponding to the global minimum obtained with potassium. The comparison with its harmonic vibrational spectrum (Figure S14) confirms the expected interaction scheme. Note that globally, IRMPD spectra obtained for the $\mathrm{Cs}^{+}-$ complexes are less informative than those obtained in presence of $\mathrm{K}^{+}$, due to a much higher photofragmentation yield (the unique photofragment being $\mathrm{Cs}^{+}$) for the same irradiation time, resulting in an overwhelming signal around $1325 \mathrm{~cm}^{-1}$, and small bands that were observed for the $\mathrm{K}^{+}$complexes (Figure 9) are no longer detected. One can observe that all the experimental bands can be interpreted by the computed vibrational spectra (see Table S3 and S4 for the assignment of the experimental bands). The high photofragmentation yield observed for the $\mathrm{Cs}+/$ complex, and the slight blue shift observed for the $\mathrm{C} 2=\mathrm{O} 2$ stretching mode when replacing $\mathrm{K}^{+}$by $\mathrm{Cs}^{+}$, indicate the stronger affinity of $\mathrm{K}^{+}$ions for the ligand. This was rather expected because in the gas phase potassium cation affinities (KCA) are higher than cesium cation affinities (CsCA) for ligands with carbonyl groups. ${ }^{15}$ This is presently confirmed by the estimate of the thermochemical data as shown in Table 2 (entry 4). Nonetheless, the calculated deformation energies are for the $\mathrm{Cs}^{+}$ complexes are small (Table 3). The lower interaction energies are thus essentially due to the larger size of the cation that cannot interact strongly with the sulfur and $\mathrm{Ph}_{1}$ ring (Table 1).

\section{Conclusions}

In summary, the ability of (dihydro)naphthothiophenes to host alkali metals is described. The molecular platform was functionalized to comprise one $\pi$ fragment, one carbonyl moiety and a central sulfur atom which define an electronically rich bay region. Those key fragments have been topologically arranged so has to provide a favourable electronic density for the elaboration of complexes with alkali cations. In this communication, four complexes combining one rigid or one flexible naphthothiophenes ligand and two cations $\left(\mathrm{K}^{+}\right.$and $\left.\mathrm{Cs}^{+}\right)$ have been studied. The computational study of both the ligands and their corresponding complexes has been carried out. For both flexible (4) and rigid (1) platforms, $\mathrm{K}^{+}$complexes display highest binding energies with respect to the $\mathrm{Cs}^{+}$analogues. In all cases, the overlap of the ligand and the corresponding $\mathrm{K}^{+}$- and $\mathrm{Cs}^{+}$-complexes evidenced very slight deformation of the ligand topology during the binding process. IRMPD experimental data gave useful insights for the detailed characterization of all complexes and are in good agreement with computed vibrational spectra. The theoretical analysis evidenced that the formation of alkali metal complexes arises from a deep cooperative intervention of $\mathrm{S}^{-}, \mathrm{CO}^{-}$and $\pi$-interactions. 


\section{Experimental Section}

\section{General Experimental Procedures and Materials}

Unless otherwise noted, all starting materials were obtained from commercial suppliers and used without purification. Petroleum ether was distilled under argon, THF was dried over sodium/benzophenone and distilled under argon. Reaction progress was carried out using E. Merck silica gel 60 F245 precoated aluminium plates $(0.25 \mathrm{~mm})$. Gerudan Si 60 silica gel $60(40-63 \mu \mathrm{m})$ was used for flash chromatography.

\section{Instrumentation}

${ }^{1} \mathrm{H}$ NMR spectra were recorded with Brüker AV-I $300 \mathrm{MHz}$ spectrometer at $298 \mathrm{~K}$, referenced to tetramethylsilane signal and were calibrated using residual protium in $\mathrm{CDCl}_{3}(\delta=7.26 \mathrm{ppm}$ ) according to the litterature ${ }^{1} .{ }^{13} \mathrm{C}$ NMR spectra were recorded with Brüker AV-I $300 \mathrm{MHz}$ spectrometer at $75 \mathrm{MHz}$ at $298 \mathrm{~K}$, and were calibrated using $\mathrm{CDCl}_{3}(\delta=$ $77.16 \mathrm{ppm})^{1}$. ${ }^{1} \mathrm{H}$ NMR spectroscopic data are reported as follows: chemical shift $\delta$ [parts per million] (multiplicity, coupling constant in Hertz $[\mathrm{Hz}]$, integration). Multiplicities are reported as follows: $\mathrm{s}=$ singlet, $\mathrm{d}=$ doublet, $\mathrm{t}=$ triplet, $\mathrm{dd}=$ doublet of doublets, $\mathrm{m}=$ multiplet. ${ }^{13} \mathrm{C} \mathrm{NMR}$ spectroscopic data are reported in terms of chemical shifts $\delta$ in parts per million [ppm]. High resolution mass spectra (HRMS) were obtained with a Waters Xevo QTOF instrument with an electrospray ionization source (ESI+), using Leucine Enkephaline solution as internal calibrant. UPLC/ESI+ analyses were performed on a Waters Xevo QTOF instrument with $\mathrm{ESI}+$ detector and the achiral stationary phase Waters UPLC C18. The eluent is: solvent $A-\mathrm{H}_{2} \mathrm{O}+0.1 \% \mathrm{HCOOH}$, solvent $\mathrm{B}-$ $\mathrm{ACN}+0.1 \% \mathrm{HCOOH}, \mathrm{A} / \mathrm{B} 95 / 5$ during 0.5 min then gradient $\mathrm{A} / \mathrm{B} 95 / 5$ to 0/100 during $4.5 \mathrm{~min}, 0450 \mathrm{~mL} \cdot \mathrm{min}^{-1}$. Samples were solubilized in ACN at $1 \mathrm{mg} / \mathrm{mL}$ and then diluted by 1000 .

\section{Methyl 9-bromo-4,5-dihydronaphtho[1,2-b]thiophene-2-carboxylate} (2):

$\mathrm{N}, \mathrm{N}$-Dimethylformamide $(2.50 \mathrm{~mL}, 37.59 \mathrm{mmol}, 8 \mathrm{eq}$.) was slowly added to a well-stirred and cooled solution of phosphoryl trichloride $(2.63 \mathrm{~mL}$, $28.22 \mathrm{mmol}, 7$ eq.) at $0{ }^{\circ} \mathrm{C}$. As soon as the reaction mixture precipitates 8-bromo-3,4-dihydronaphthalen-1(2H)-one (907.9 mg, $4.03 \mathrm{mmol}$ ) was added to the round bottom flask diluted in a small amount of $N, N$ dimethylformamide. The ice bath was removed and the reaction mixture was allowed to warm up to room temperature and then heated at $70{ }^{\circ} \mathrm{C}$ for 2 hours. The mixture was diluted with ethyl acetate and poured into crushed ice. The aqueous layer was extracted with EtOAc. The combined organic layers were successively washed with an aqueous solution of satured sodium bicarbonate, brine and dried over $\mathrm{MgSO}_{4}$. The solvent was removed under vacuum. The crude product was purified by flash chromatography on silica gel eluting with petroleum ether/ethyl acetate (98:2) to give 8-bromo-1-chloro-3,4-dihydronaphthalene-2carbaldehyde intermediate as yellow crystals with a quantitative yield. Rf: 0,34 in petroleum ether/ethyl acetate (95:5). M. p.: $52-53^{\circ} \mathrm{C}$. ${ }^{1} \mathrm{H}$ NMR $\left(300 \mathrm{MHz}, \mathrm{CDCl}_{3}\right) \delta 10.34(\mathrm{~s}, 1 \mathrm{H}), 7.53$ (dd, $\left.J=7.8,1.4 \mathrm{~Hz}, 1 \mathrm{H}\right), 7.17-$ $7.15(\mathrm{~m}, 1 \mathrm{H}), 7.14-7.07(\mathrm{~m}, 1 \mathrm{H}), 2.69-2.65(\mathrm{~m}, 2 \mathrm{H}), 2.47-2.42(\mathrm{~m}, 2$ H) ppm. ${ }^{13} \mathrm{C}$ NMR $\left(75 \mathrm{MHz}, \mathrm{CDCl}_{3}\right) \delta 190.4,144.3,144.0,137.0,134.6$, 132.3, 131.2, 126.8, 121.7, 29.3, 21.3 ppm. HRMS (ESI ${ }^{+}$.TOF) for $\mathrm{C}_{11} \mathrm{H}_{9} \mathrm{BrClO},[\mathrm{M}+\mathrm{H}]^{+}$calculated $\mathrm{m} / \mathrm{z}=270.9525$, found $\mathrm{m} / \mathrm{z}=270.9536$. To the latter intermediate $(203.6 \mathrm{mg}, 0.75 \mathrm{mmol})$ in pyridine $(860 \mu \mathrm{L}, 10$ mmol, $14 \mathrm{eq})$ was added methyl thioglycolate $(77 \mu \mathrm{L}, 0.86 \mathrm{mmol}, 1.15$ eq) followed by triethylamine $(160 \mu \mathrm{L}, 1.2 \mathrm{mmol}, 1.6 \mathrm{eq})$. This mixture was heated at $60{ }^{\circ} \mathrm{C}$ for two hours and then left to cool down to room temperature. An aqueous solution of $50 \%(w / w)$ potassium hydroxide $(165 \mu \mathrm{L})$ was added and mixture obtained was stirred for 20 more minutes. The medium was poured over ice and rinsed with dichloromethane, before adding dropwise a solution of hydrochloric acid $(20 \mathrm{~mL}, 2 \mathrm{M})$. The organic layer was extracted and washed with successively a solution of hydrochloric acid $(20 \mathrm{~mL}, 2 \mathrm{M})$, a solution of sodium hydroxide $(10 \mathrm{~mL}, 2 \mathrm{M})$ and water. The organic layers were dried over $\mathrm{MgSO}_{4}$, filtered and concentrated under reduce pressure to yield methyl 9-bromo-4,5-dihydronaphtho[1,2-b]thiophene-2-carboxylate 2 as a pale yellow solid (231.6 mg, $0.73 \mathrm{mmol}, 98 \%)$. Rf : 0,21 in petroleum ether/ethyl acetate (95:5). M. p.: $97-98^{\circ} \mathrm{C} .{ }^{1} \mathrm{H}$ NMR $\left(300 \mathrm{MHz}, \mathrm{CDCl}_{3}\right)$ $\delta 7.57(\mathrm{~s}, 1 \mathrm{H}), 7.45(\mathrm{~d}, J=7.8 \mathrm{~Hz}, 1 \mathrm{H}), 7.13(\mathrm{~d}, J=7.8 \mathrm{~Hz}, 1 \mathrm{H}), 6.95$ (t, $J=8.0 \mathrm{~Hz}, 1 \mathrm{H}), 3.86(\mathrm{~s}, 3 \mathrm{H}), 2.89-2.84(\mathrm{~m}, 2 \mathrm{H}), 2.75-2.70(\mathrm{~m}, 2 \mathrm{H})$ ppm. ${ }^{13} \mathrm{C}$ NMR $\left(75 \mathrm{MHz}, \mathrm{CDCl}_{3}\right) \delta 163.2,140.9,140.1,139.5,133.0$, $132.4,131.4,131.1,128.5,127.6,120.3,52.3,30.9,24.2$ ppm. HRMS (ESI ${ }^{+}$-TOF) for $\mathrm{C}_{14} \mathrm{H}_{12} \mathrm{BrO}_{2} \mathrm{~S},[\mathrm{M}+\mathrm{H}]^{+}$calculated $\mathrm{m} / \mathrm{z}=322.9741$, found $m / z=322.9745$.

\section{Methyl 9-bromonaphtho[1,2-b]thiophene-2-carboxylate (3):}

Methyl 9-bromo-4,5-dihydronaphtho[1,2-b]thiophene-2-carboxylate 2 (620.1 mg, $1.92 \mathrm{mmol}$ ) and DDQ (542 mg, $2.40 \mathrm{mmol}, 1.2$ eq.) were dissolved in dry toluene $(11 \mathrm{~mL})$ and heated under reflux overnight. The mixture was cooled to room temperature and concentrated under vaccum. The crude product was dissolved in methylene chloride and washed several times with a saturated solution of $\mathrm{NaHCO}_{3}$ and brine. The organic layers were dried over anhydrous $\mathrm{MgSO}_{4}$, filtered and concentrated under reduced pressure. The crude product was purified by flash chromatography on silica gel column eluting with petroleum ether/EtOAc (95:5) to afford methyl 9-bromonaphtho[1,2-b]thiophene-2-carboxylate 3 as a white solid (468.7 mg, $1.46 \mathrm{mmol}, 76 \%$ ). Rf : 0,24 in petroleum ether/ethyl acetate (95:5). M. p.: $140-141{ }^{\circ} \mathrm{C} .{ }^{1} \mathrm{H}$ NMR $(300 \mathrm{MHz}$, $\left.\mathrm{CDCl}_{3}\right) \delta 8.23(\mathrm{~s}, 1 \mathrm{H}), 7.97$ (dd, $\left.J=4.5,1.2 \mathrm{~Hz}, 1 \mathrm{H}\right), 7.94(\mathrm{dd}, J=4.5$, $1.2 \mathrm{~Hz}, 1 \mathrm{H}), 7.88(\mathrm{~d}, J=8.6 \mathrm{~Hz}, 1 \mathrm{H}), 7.79(\mathrm{~d}, J=8.6 \mathrm{~Hz}, 1 \mathrm{H}), 7.41(\mathrm{t}, J$ $=7.7 \mathrm{~Hz}, 1 \mathrm{H}) \mathrm{ppm} .{ }^{13} \mathrm{C} \mathrm{NMR}\left(75 \mathrm{MHz}, \mathrm{CDCl}_{3}\right) \delta 163.4,139.5,139.0$, 134.6, 133.6, 132.6, 130.4, 129.0, 128.7, 127.1, 126.7, 124.0, 120.3, 52.6 ppm. HRMS (ESI ${ }^{+}-\mathrm{TOF}$ ) for $\mathrm{C}_{14} \mathrm{H}_{9} \mathrm{BrO}_{2} \mathrm{~S}$, [M] ${ }^{+\cdot}$ calculated $\mathrm{m} / \mathrm{z}=$ 319.9507 , found $m / z=319.9508$.

\section{Typical procedure for Suzuki-Miyaura coupling :}

To a stirred suspension of 9-bromodihydronaphthothiophene or 9bromonaphthothiophene, phenyl boronic acid (2 eq.) and cesium fluoride ( 4 eq.) in dry and degassed THF, were added palladium acetate (10 $\mathrm{mol} \%$ ) and SPhos (20 mol \%). The mixture was stirred under reflux of THF until completion, determined by TLC analysis. The solution was let to return to room temperature, diluted with water and then extracted with methylene chloride. The combined organic layers were dried over anhydrous magnesium sulfate, filtered, and concentrated under reduced pressure. The crude product was purified by flash chromatography on silica gel eluting with the corresponding solvent mixture to give the desired product.

\section{Methyl 9-phenylnaphtho[1,2-b]thiophene-2-carboxylate (1):}

Typical procedure was applied using methyl 9-bromonaphtho[1,2 b]thiophene-2-carboxylate 3 (200 $\mathrm{mg}, 0,62 \mathrm{mmol})$ and phenylboronic acid $(150,6 \mathrm{mg}, 1,24 \mathrm{mmol})$ to give as methyl 9-phenylnaphtho[1,2b]thiophene-2-carboxylate as a white solid (193,1 mg, 0,60 mmol, $97 \%$ ) after purification on silica gel elution with petroleum ether/EtOAc (98:2). Rf: 0,51 in petroleum ether/ethyl acetate (9:1). M. p.: $101-102{ }^{\circ} \mathrm{C} .{ }^{1} \mathrm{H}$ NMR $\left(300 \mathrm{MHz}, \mathrm{CDCl}_{3}\right) \delta 8.07(\mathrm{~s}, 1 \mathrm{H}), 7.98(\mathrm{dd}, J=1.4,8.1 \mathrm{~Hz}, 1 \mathrm{H})$, 7.84 (s, $2 \mathrm{H}$ ), 7.63-7.53 (m, $4 \mathrm{H}), 7.48-7.41$ (m, $3 \mathrm{H}), 3.84$ (s, $3 \mathrm{H}) \mathrm{ppm}$. ${ }^{13} \mathrm{C}$ NMR $\left(75 \mathrm{MHz}, \mathrm{CDCl}_{3}\right) \delta 163.4,140.8,140.4,139.4,138.1,133.3$, $132.9,130.3,129.2,128.9,128.9,128.9,127.6,126.9,125.9,123.2$, 
52.4 ppm. HRMS (ESI ${ }^{+}$-TOF) for $\mathrm{C}_{20} \mathrm{H}_{15} \mathrm{O}_{2} \mathrm{~S},\left[\mathrm{M}+\mathrm{H}^{+}\right.$calculated $\mathrm{m} / \mathrm{z}=$ 319.0793 , found $m / z=319.0798$.

\section{Methyl 9-phenyl-4,5-dihydronaphtho[1,2-b]thiophene-2-carboxylate} (4):

Typical procedure was applied using methyl 9-bromo-4,5dihydronaphtho[1,2-b]thiophene-2-carboxylate $2(107 \mathrm{mg}, 0.33 \mathrm{mmol})$ and phenylboronic acid $(80.8 \mathrm{mg}, 0.66 \mathrm{mmol})$ to give methyl 9-phenyl4,5-dihydronaphtho[1,2-b]thiophene-2-carboxylate as a white solid (99.3 $\mathrm{mg}, 0.31 \mathrm{mmol}, 94 \%$ ) after purification on silica gel column eluting with pentane/EtOAc (99:1). Rf : 0,43 in petroleum ether/ethyl acetate (9:1). M. p.: $133-134{ }^{\circ} \mathrm{C} .{ }^{1} \mathrm{H}$ NMR $\left(300 \mathrm{MHz}, \mathrm{CDCl}_{3}\right) \delta 7.49(\mathrm{~s}, 1 \mathrm{H}), 7.46-7.44$ $(\mathrm{m}, 2 \mathrm{H}), 7.32-7.20(\mathrm{~m}, 3 \mathrm{H}), 7.11(\mathrm{dd}, J=2.0,7.0 \mathrm{~Hz}, 1 \mathrm{H}), 3.76(\mathrm{~s}, 3 \mathrm{H})$ 3.01-2.96 (m, $2 \mathrm{H}), 2.86-2.81(\mathrm{~m}, 2 \mathrm{H}) \mathrm{ppm} .{ }^{13} \mathrm{C}$ NMR $\left(75 \mathrm{MHz}, \mathrm{CDCl}_{3}\right)$ $\delta 163.8,142.4,141.5,140.1,139.8,137.8,132.95,132.0,130.7,130.1$, $130.0,129.5,129.1,128.2,128.0,52.5,30.8,24.7$ ppm. HRMS $\left(\mathrm{ESI}^{+}{ }_{-}\right.$ TOF) for $\mathrm{C}_{20} \mathrm{H}_{17} \mathrm{O}_{2} \mathrm{~S},[\mathrm{M}+\mathrm{H}]^{+}$calculated $\mathrm{m} / \mathrm{z}=321.0949$, found $\mathrm{m} / \mathrm{z}=$ 321.0945.

\section{Acknowledgements}

This work was supported by the French National Research Agency under the Program Investing in the Future Grant $n^{\circ}$ ANR10-IDEX-0003-02 for a PhD fellowship to $\mathrm{HB}$, and by CHARMMMAT ANR-11-LABX-0039-grant for a post-doctoral fellowship to SP.

The authors wish to thank the CLIO team (J. M. Ortega, C. Six, G. Perilhous, J. P. Berthet) as well as P. Maître and V. Steinmetz for their support during the experiments.

Keywords: weak interaction $\bullet$ bay region $\cdot$ alkali metal complexes • thiophenes

[1] a) L. M. Salonen, M. Ellermann, F. Diederich, Angew. Chem. Int. Ed. 2011, 50, 4808-4842; b) R. K. Raju, J. W. G. Bloom, Y. An, S. E. Wheeler, ChemPhysChem 2011, 12, 3116-3130; c) An special issue on "Aromatic Interactions in Chemistry and Biology"was recently publised in the journal Acc. Chem. Res. 2013, 46, 873-1050; d) S. E. Wheeler, Acc. Chem. Res. 2013, 46, 1029-1038; e) S. Li, Y. Xu, Q. Shen, X. Liu, J. Lu, Y. Chen, T. Lu, C. Luo, X. Luo, M. Zheng, H. Jiang Curr. Pharm. Des. 2013, 19, 6522-6533; f) M. Alonso, T. Woller, F. J. Martin-Martinez, J. Contreras-Garcia, P. Geerlings, F. De Proft, Chem. Eur. J. 2014, 20, 4931-4941.

[2] a) A. Star, T.-R. Han, J.-C. P. Gabriel, K. Bradley, G. Grüner, Nano Lett. 2003, 3, 1421-1423; b) S. M. Kozlov, F. Viñes, A. Görling, Carbon 2012, 50, 2482-2492; c) M. Kumar, K. V. Rao, S. J. George, Phys. Chem. Chem. Phys. 2014, 16, 1300-1313; d) B. A. Ikkanda, B. L. Iverson, Chem. Commun. 2016, 52, 7752-7759; e) T. Hüffer, H. Sun, J. D. Kubicki, T. Hofmann, M. Kah, Environ. Sci.: Nano 2017, 4, 1045 1053; f) M. Ben Yahia, M. Tounsi, F. Aouaini, S. Knani, M. Ben Yahia, A. Ben Lamine, RSC Adv. 2017, 7, 44712-44723.

[3] a) L. Pu, J. Pharmacol and Photobiol. A: Chemistry 2003, 155, 47-55 b) K. Takaishi, M. Kawamoto, Molecules 2011, 16, 1603-1624; c) D Xiao, Z. Zhang, X. Zhang, Org. Lett. 1999, 1, 1679-1681.

[4] a) G. Pieters, V. Terrasson, A. Gaucher, D. Prim, J. Marrot, Eur. J. Org. Chem. 2010, 30, 5800-5806; b) D. K. Judge, P. Haycock, R. D. Richardson, M. J. Fuchter, Synlett 2013, 24, 2365-2369.
[5] a) C. Wolf, X. Mei, J. Am. Chem. Soc. 2003, 125, 10651-10658; b) X Mei, R. M. Martin, C. Wolf, J. Org. Chem. 2006, 71, $2854-2861$; c) G. Pieters, A. Gaucher, D. Prim, J. Marrot, Chem. Commun. 2009, 48274828; d) G. Pieters, A. Gaucher, J. Marrot, F. Maurel, J.-V. Naubron, M. Jean, N. Vanthuyne, J. Crassous, D. Prim, Org. Lett. 2011, 13, 44504453

[6] X.-Y. Wang, A. Narita, W. Zhang, X. Feng, K. Müllen, J. Am. Chem Soc. 2016, 138, 9021-9024.

[7] G. Pieters, A. Gaucher, S. Marque, F. Maurel, P. Lesot, D. Prim, J. Org Chem. 2010, 75, 2096-2098.

[8] a) A. Bermejo, A. Ros, R. Fernández, J. M. Lassaletta J. Am. Chem. Soc. 2008, 130, 15798-15799; b) A. Ros, B. Estepa, P. Ramírez-López, E. Álvarez, R. Fernández, J. M. Lassaletta, J. Am. Chem. Soc. 2013, $135,15730-15733$

[9] a) S.K. Pal, T. Sahu, T. Misra, T. Ganguly, T.K. Pradhan, A. De, J. Photochem. Photobiol. A: Chemistry 2005, 174, 138-148; b) S. K. Pal S. Bhattacharaya, S. K. Batabyal, T. K. Pradhan, T. Ganguly, J. Photochem. Photobiol. A: Chemistry 2007, 189, 86-93; c) T. Dutta, Y. Li, A. L. Thornton, D.-M. Zhu, Z. Peng, J. Polymer Science Part A Polymer Chemistry 2013, 51, 3818-3828;

[10] a) E. S. Meadows, S. L. De Wall, L. J. Baarbour G. W. Gokel, J. Am Chem. Soc. 2001, 123, 3092; b) J. P. G. Dougherty, A. Denis, Proc. Natl. Acad. Sci. USA, 1999, 96, 9459-9464; c) Y. Hagiwara, H. Matsumura, M. Tateno, J. Am. Chem. Soc., 2009, 131, 16697-16705.

[11] G. Bernard, M. I. Shevell, Pediatr. Neurol. 2008, 38, 73-85.

[12] a) M. T. Rodgers, P. B. Armentrout, Chem. Rev. 2016, 116, 5642-5687; b) B. E. Ziegler, M. Lecours, R. A. Marta, J. Featherstone, E. Fillion, W S. Hopkins, V. Steinmetz, N. S. Keddie, D. O'Hagan, T. B. MacMahon, J. Am. Chem. Soc. 2016, 138, 7460-7463; c) E. S. Meadows, S. L. De Wall, L. J. Barbour, G. W. Gokel, J. Am. Chem. Soc., 2001, 123, 3092 3107

[13] a) T. W. Craven, M. K. Cho, N. J. Traaseth, J. Am. Soc. 2016, 138, 1543-1550; b) J. C. Ma, D. A. Dougherty, Chem. Rev. 1997, 97, 1303 1324

[14] a) H. Boufroura, A. Souibgui, A. Gaucher, J. Marrot, G. Pieters, F. Aloui, B. Ben Hassine, G. Clavier, D. Prim, Org. Biomol. Chem. 2015, 13 10844-10851; b) A. Souibgui, A. Gaucher, J. Marrot, F. Aloui, F. Mahuteau-Betzer, B. Ben Hassine, D. Prim, Eur. J. Org. Chem. 2013 4515-4522.

[15] B. R. Beno, K. S. Yeung, M. D. Bartberger, L. D. Pennington, N. A Meanwell, J. Med. Chem. 2015, 58, 4383-4438.

[16] a) T. D. Fridgen, Mass Spectrom. Rev. 2009, 28, 586-607; b) B Chiavarino, M.-E. Crestoni, S. Fornarini, D. Scuderi, J-Y. Salpin, Inorg Chem. 2015, 54, 3513-3522; c) B. E. Ziegler, M. Lecours, R. A. Marta J. Featherstone, E. Fillion, W. S. Hopkins, V. Steinmetz, N. S. Keddie, D. O'Hagan, T. B. McMahon, J. Am. Chem. Soc. 2016, 138, 7460-7463 d) B. Chiavarino, M.-E. Crestoni, S. Fornarini, D. Scuderi, J-Y. Salpin , Inorg. Chem. 2017, 56, 8793-8801.

[17] a) A. D. Becke, J. Chem. Phys. 1993, 98, 5648-5652; b) C. T. Lee, W. T. Yang, R. G. Parr, Phys. Rev. B 1988, 37, 785-789.

[18] Y. Zhao, D. G. Truhlar, Theor. Chem. Acc. 2008, 120, 215-241.

[19] the deformation energy corresponds to the energy difference between the structure of the ligand within the complex and the structure of the free ligand.

[20] P. J. Hay, W. R. Wadt, J. Chem. Phys. 1985, 82, 299-310. 
Entry for the Table of Contents (Please choose one layout)

\section{Layout 2:}

\section{FULL PAPER}

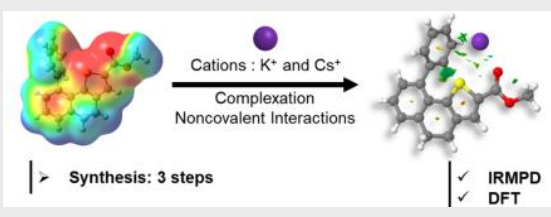

The cooperativity of three non-covalent interactions within a well-defined electronically rich bay region of the ligand allowed complexation of alkali metals.
Hamza Boufroura, Salomé Poyer, Anne Gaucher, Cécile Huin, Jean-Yves Salpin, Gilles Clavier, and Damien Prim*

Page No. - Page No.

Topologic and electronic density driven generation of alkali cation complexes 